\title{
STUDY OF A LOGISTIC EQUATION WITH LOCAL AND NON-LOCAL REACTION TERMS
}

\author{
Manuel Delgado - Giovany M. Figueiredo \\ Marcos T.O. Pimenta - Antonio SuÁrez
}

(Submitted by J. Mawhin)

\begin{abstract}
We examine a logistic equation with local and non-local reaction terms both for time dependent and steady-state problems. Mainly, we use bifurcation and monotonicity methods to prove the existence of positive solutions for the steady-state equation and sub-supersolution method for the long time behavior for the time dependent problem. The results depend strongly on the size and sign of the parameters on the local and non-local terms.
\end{abstract}

\section{Introduction}

In this paper we study the non-local parabolic problem

$$
\begin{cases}u_{t}-\Delta u=u\left(\lambda+b \int_{\Omega} u^{r} d x-u\right) & \text { in } \Omega \times(0, \infty), \\ u=0 & \text { on } \partial \Omega \times(0, \infty), \\ u(x, 0)=u_{0}(x) \geq 0 & \text { in } \Omega,\end{cases}
$$

2010 Mathematics Subject Classification. Primary: 35R09, 45K05; Secondary: 35J60, $35 \mathrm{~K} 35,35 \mathrm{~A} 25$.

Key words and phrases. Logistic equation; local and non-local terms; bifurcation methods.

M. Delgado and A. Suárez were supported by FEDER and Ministerio de Economía y Competitividad (Spain) under grant under grant MTM2012-31304.

G. Figueiredo was supported by CNPQ/PQ 301242/2011-9 and 200237/2012-8.

M. Pimenta was supported by FAPESP, Brazil, 2012/20160-0 and 2014/16136-1 and CNPQ 442520/2014-0. 
and the corresponding steady-state problem

$$
\begin{cases}-\Delta u=u\left(\lambda+b \int_{\Omega} u^{r} d x-u\right) & \text { in } \Omega, \\ u=0 & \text { on } \partial \Omega,\end{cases}
$$

where $\Omega \subset \mathbb{R}^{N}$ is a bounded and smooth domain, $\lambda, b \in \mathbb{R}, r>0$ and $u_{0}$ is a regular positive function. In (1.1), $u(x, t)$ represents the density of a species in time $t>0$ and a habitat surrounded by inhospitable areas at the point $x \in \Omega$. Here, $\lambda$ is the growth rate of species, the term $-u$ describes the limiting effect of crowding in the population, that is, the competition of individuals of species for resources of the environment. In (1.1) we have included a non-local term with different meanings. When $b<0$ we are assuming that this limiting effect depends not only on the value of $u$ at the point $x$, but on the value of $u$ in the whole domain. When $b>0$ individuals cooperate globally to survive. When $b=0,(1.1)$ is the classical logistic equation.

Observe that when $b>0$, problem (1.1) can be regarded as a superlinear indefinite problem with non-local superlinear term, similar to the classical superlinear problem

$$
\begin{cases}u_{t}-\Delta u=u\left(\lambda+b a^{+} u^{r}-a^{-} u^{r}\right) & \text { in } \Omega \times(0, \infty), \\ u=0 & \text { on } \partial \Omega \times(0, \infty), \\ u(x, 0)=u_{0}(x) \geq 0 & \text { in } \Omega,\end{cases}
$$

where $a \in C^{1}(\bar{\Omega}), a^{+}:=\max \{a(x), 0\}, a^{-}:=\max \{-a(x), 0\}$. The latter has been studied in detail in [14], [15], [17], see also references therein. This class of local problems has been considered also with other boundary conditions, for example, non-homogeneous Dirichlet boundary conditions, see [9] and [18], where multiplicity results are shown. We do not consider the non-local counterpart in this paper.

The introduction of non-local terms in the equation and in the boundary conditions has shown to be useful for modelling a number of processes in different fields such as mathematical physics, mechanics of deformable solids, mathematical biology and many others. For examples of its application in population dynamics, see, for instance, [8], [7] and [11].

Let us summarize our main results. Denote by $\lambda_{1}$ the principal eigenvalue of the Laplacian subject to homogeneous Dirichlet boundary conditions and by $\varphi_{1}$ the positive eigenfunction associated to $\lambda_{1}$ such that $\left\|\varphi_{1}\right\|_{\infty}=1$.

Regarding parabolic problem (1.1), first we prove the existence and uniqueness of positive local in time solution. Next, we analyze the long time behaviour of the solution. In particular:

(1) If $b<0$ the solution of (1.1) is global in time and bounded. Moreover, the solution goes to zero as $\lambda<\lambda_{1}$. 
(2) Assume now $b>0$.

(a) The trivial solution is locally exponentially stable for $\lambda<\lambda_{1}$, that is, for small enough $u_{0}$ the solution goes to zero if $t \rightarrow \infty$.

(b) If $r<1$ or $r=1$ and $b$ is small enough, the solution of (1.1) is global in time and bounded. Moreover, the solution goes to zero if $\lambda$ is small enough.

(c) If $r>1$ or $r=1$ and $b$ is large enough, the solution of (1.1) blows up in finite time for $\lambda$ or $u_{0}$ large enough.

We refer to Section 6 for more specific results. We would like to remark that similar results have been obtained in [21], [22] and [19], see also references therein, for the problem

$$
u_{t}-\Delta u=\int_{\Omega} u^{r}(x, t) d x-k u^{p}, \quad \text { for } r, p \geq 1 \text { and } k \geq 0 .
$$

Regarding problem (1.2). The case $b<0$ and $r=1$ has been analyzed in [23], where the existence and uniqueness of positive solution of (1.2) were established. We improve these results considering all the cases for $r>0$. The case $b=0$ (the pure local model) is well-known, see Proposition 2.1. The equation

$$
-\Delta u=u\left(\lambda+b \int_{\Omega} u d x+u\right)
$$

with $b<0$ has been analyzed in [8].

In order to prove our results, we use mainly the bifurcation method, applied previously in this context by [1], [5] and [12].

First, we show that from the trivial solution $u=0$ an unbounded continuum of positive solutions of (1.2) emanates at $\lambda=\lambda_{1}$. Next, we study the local and global behaviour of this continuum. In particular, when $b \leq 0$ the behaviour does not depend on $r$ and we show the following result (see Figure 1 (a)).

TheOrem 1.1. Assume $b \leq 0$. Then a positive solution exists if and only if $\lambda>\lambda_{1}$. Moreover, if it exists it is unique (denote it by $\emptyset_{\lambda, b}$ ) and

$$
\lim _{b \rightarrow-\infty}\left\|\phi_{\lambda, b}\right\|_{\infty}=0
$$

When $b>0$ the behaviour depends on the size of $r$. When $r<1$ we obtain (see Figure $1(\mathrm{c})$ ) the following result.

Theorem 1.2. Assume $b>0$ and $r<1$. There exists $\lambda_{*}<\lambda_{1}$ such that (1.2) possesses at least one positive solution if and only if $\lambda \geq \lambda_{*}$. Moreover,

$$
\lim _{b \rightarrow 0^{+}} \lambda_{*}(b)=\lambda_{1} \quad \text { and } \quad \lim _{b \rightarrow+\infty} \lambda_{*}(b)=-\infty .
$$

When $r>1$ (see Figure 1(d)) we have 


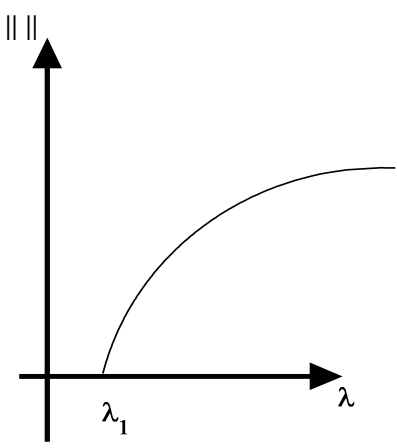

(a)

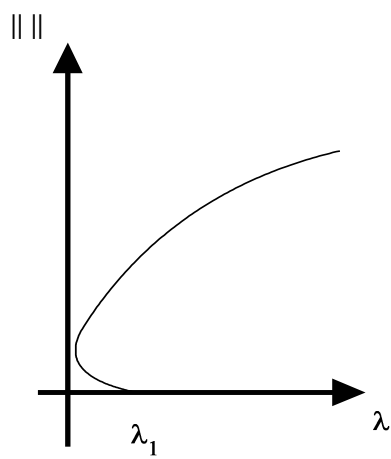

(c)

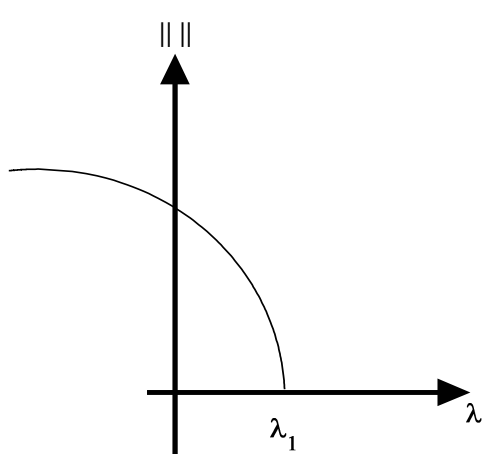

(b)

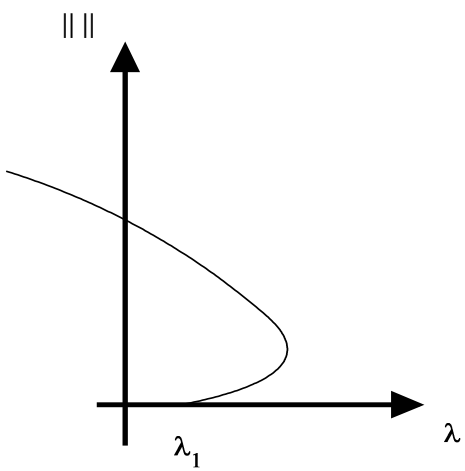

(d)

Figure 1. Bifurcation diagrams for equation (1.2).

Theorem 1.3. Assume $b>0$ and $r>1$. There exists $\lambda^{*}>\lambda_{1}$ such that (1.2) possesses at least one positive solution if and only if $\lambda \leq \lambda^{*}$. Moreover,

$$
\lim _{b \rightarrow 0^{+}} \lambda^{*}(b)=+\infty \quad \text { and } \quad \lim _{b \rightarrow+\infty} \lambda^{*}(b)=\lambda_{1} .
$$

Let us remark that, unlike in the local case, we do not need to impose any restrictions on $r$ in order to get the a priori bounds. Indeed, if we were considering the local case

$$
-\Delta u=u\left(\lambda+b u^{r}-u\right),
$$

then in order to obtain a priori bounds, we require $r+1<(N+2) /(N-2)$, see [10].

Finally, in the case $r=1$, the behaviour depends of the size of $b$ :

Theorem 1.4. Assume $b>0$ and $r=1$.

(a) Assume that $b<1 /|\Omega|$. Then, there exists a positive solution for $\lambda>\lambda_{1}$. 
(b) Assume that $b>1 / \int_{\Omega} \varphi_{1} d x$. Then, there exists a positive solution if and only if $\lambda<\lambda_{1}$.

Here $|\Omega|$ stands for the measure of $\Omega$. When $b$ is small enough, the bifurcation is similar to the case $b \leq 0$ (see Figure 1 (a)), whereas when $b$ is large enough we have a positive solution for $\lambda<\lambda_{1}$ (see Figure 1(b)).

There exists a gap in our results for $b \in\left(1 /|\Omega|, 1 / \int_{\Omega} \varphi_{1}\right)$. In this case, we know that there exists an unbounded continuum of positive solutions bifurcating from $(\lambda, u)=\left(\lambda_{1}, 0\right)$, moreover, we know its local bifurcation direction (see Theorem 2.2), but we are not able to assure the global behaviour of the continuum. Observe, that this does not occur in the homogeneous Neumann case. Indeed, in this case $\lambda_{1}=0$ and $\varphi_{1}=1$. Hence, $1 /|\Omega|=1 / \int_{\Omega} \varphi_{1}$ and for $b=1 /|\Omega|$ there exist infinite positive solutions for $\lambda=\lambda_{1}=0$.

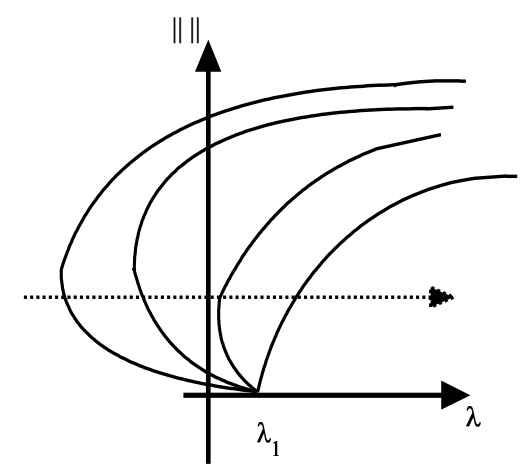

(a)

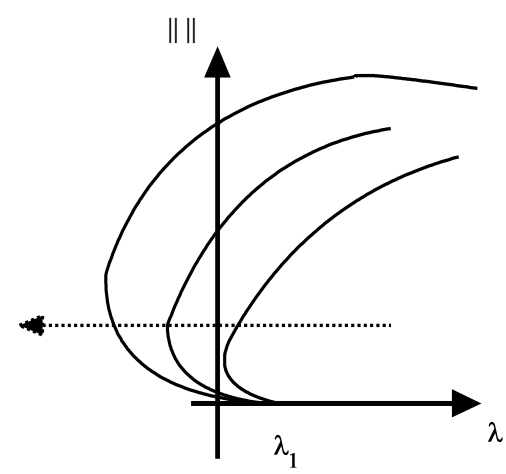

(c)

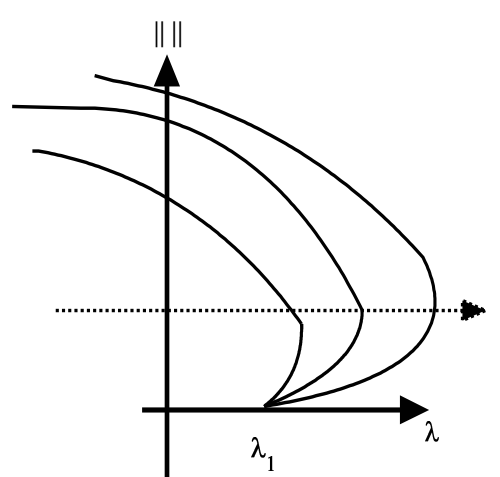

(b)

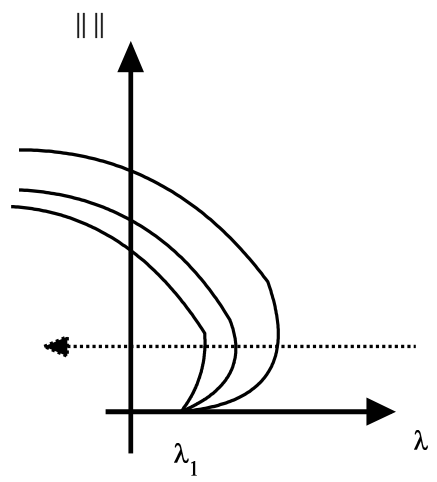

(d)

Figure 2. Bifurcation diagrams for equation (1.2) moving $b$.

Look now at Figure 2, where we have presented different bifurcation diagrams moving the parameter $b$. Figures 2 (a) and (b) depict the bifurcation diagrams 
when $b \rightarrow 0$ in the cases $r<1$ and $r>1$, respectively. Figures 2 (c) and (d) correspond to the case $b \rightarrow+\infty$ for $r<1$ and $r>1$.

The paper is organized as follows. In Section 2 we prove the existence of an unbounded continuum of positive solutions of (1.2). Section 3 is devoted to proving the non-existence results and a priori bounds of positive solutions of (1.2). In Section 4 we show the stability of solutions in some cases. Section 5 is dedicated to proving Theorems 1.1, 1.2, 1.3 and 1.4. Finally, in Section 6 we study parabolic problem (1.1).

\section{Bifurcation results}

We shall prove that from the trivial solution $u \equiv 0$ an unbounded continuum of positive solutions of (1.2) bifurcates at $\lambda=\lambda_{1}$.

First recall the principal results for the classical logistic equation (see Lemma 7.8 in [6] for $(2.2))$

$$
\begin{cases}-\Delta u=u(\mu-u) & \text { in } \Omega, \\ u=0 & \text { on } \partial \Omega .\end{cases}
$$

Proposition 2.1. There exists a positive solution of (2.1) if and only if $\mu>\lambda_{1}$. Moreover, it is unique if it exists (denote it by $\theta_{\mu}$ ) and the following inequalities hold:

(a)

$$
\left(\mu-\lambda_{1}\right) \varphi_{1} \leq \theta_{\mu} \leq \min \left\{\mu, K\left(\mu-\lambda_{1}\right)\right\}
$$

for some $K \geq \int_{\Omega} \varphi_{1}^{2} d x / \int_{\Omega} \varphi_{1}^{3} d x$ independent of $\mu$.

(b) If $\underline{u}>0$ is a subsolution of (2.1), then $\underline{u} \leq \theta_{\mu}$.

(c) If $\bar{u}>0$ is a supersolution of (2.1), then $\theta_{\mu} \leq \bar{u}$.

Consider the Banach space $X:=C_{0}(\bar{\Omega})$ and denote $B_{\rho}:=\left\{u \in X:\|u\|_{\infty}<\right.$ $\rho\}$. Define

$$
f(u):=u^{+}\left(\lambda+b \int_{\Omega}\left(u^{+}\right)^{r} d x-u\right),
$$

and the map $\mathcal{K}_{\lambda}: X \mapsto X$ by $\mathcal{K}_{\lambda}(u):=u-(-\Delta)^{-1}(f(u))$, where $u^{+}:=\max \{u, 0\}$ and $(-\Delta)^{-1}$ is the inverse of the operator $-\Delta$ under homogeneous Dirichlet boundary conditions. Now, it is clear that $u$ is a non-negative solution of (1.2) if and only if $u$ is a zero of the map $\mathcal{K}_{\lambda}$.

The main result of this section is:

THEOREM 2.2. The value $\lambda=\lambda_{1}$ is the only bifurcation point from the trivial solution for (1.2). Moreover, there exists a continuum $\mathcal{C}_{0}$ of non-negative solutions of (1.2) unbounded in $\mathbb{R} \times X$ emanating from $\left(\lambda_{1}, 0\right)$. Furthermore:

(a) If $b \leq 0$, the direction of bifurcation is supercritical. 
(b) Assume $b>0$.

- If $r<1$, the direction of bifurcation is subcritical.

- If $r>1$, the direction of bifurcation is supercritical.

- Assume that $r=1$ and denote

$$
b_{0}:=\frac{\int_{\Omega} \varphi_{1}^{3} d x}{\int_{\Omega} \varphi_{1} d x \int_{\Omega} \varphi_{1}^{2} d x} .
$$

If $b>b_{0}\left(\right.$ resp. $\left.b<b_{0}\right)$ the direction of bifurcation is subcritical (resp. supercritical).

Recall that we say that the direction of bifurcation is subcritical (resp. supercritical) if there exists a neighborhood $V$ of $\left(\lambda_{1}, 0\right)$ such that every solution $(\lambda, u) \in V$ satisfies $\lambda<\lambda_{1}$ (resp. $\lambda>\lambda_{1}$ ).

In order to prove this result we use the Leray-Schauder degree of $\mathcal{K}_{\lambda}$ on $B_{\rho}$ with respect to zero, denoted by $\operatorname{deg}\left(\mathcal{K}_{\lambda}, B_{\rho}\right)$, and the index of the isolated zero $u$ of $\mathcal{K}_{\lambda}$, denoted by $i\left(\mathcal{K}_{\lambda}, u\right)$.

Lemma 2.3. If $\lambda<\lambda_{1}$, then $i\left(\mathcal{K}_{\lambda}, 0\right)=1$.

Proof. Fix $\lambda<\lambda_{1}$. Define the map

$$
\mathcal{H}_{1}:[0,1] \times X \mapsto X ; \quad \mathcal{H}_{1}(t, u):=(-\Delta)^{-1}(t f(u)) .
$$

We claim that there exists $\delta>0$ such that

$$
u \neq \mathcal{H}_{1}(t, u) \quad \text { for all } u \in \bar{B}_{\delta} \backslash\{0\} \text {, and } t \in[0,1] .
$$

Indeed, suppose that there exist sequences $u_{n} \in X \backslash\{0\}$ with $\left\|u_{n}\right\|_{\infty} \rightarrow 0$ and $t_{n} \in[0,1]$ such that $u_{n}=\mathcal{H}_{1}\left(t_{n}, u_{n}\right)$, that is $-\Delta u_{n}=t_{n} f\left(u_{n}\right)$, and so $u_{n} \geq 0$. Define $w_{n}:=u_{n} /\left\|u_{n}\right\|_{\infty}$. Then,

$$
-\Delta w_{n}=t_{n} w_{n}\left(\lambda+b \int_{\Omega} u_{n}^{r} d x-u_{n}\right),
$$

and then passing to the limit $-\Delta w=t_{0} \lambda w$, for some $w \geq 0,\|w\|_{\infty}=1$, $t_{0} \in[0,1]$. Hence, $t_{0} \lambda=\lambda_{1}$, a contradiction as $\lambda<\lambda_{1}$.

Taking now $\varepsilon \in(0, \delta]$, the homotopy defined by $\mathcal{H}_{1}$ is admissible, hence

$$
\begin{aligned}
i\left(\mathcal{K}_{\lambda}, 0\right) & =\operatorname{deg}\left(\mathcal{K}_{\lambda}, B_{\varepsilon}\right)=\operatorname{deg}\left(I-\mathcal{H}_{1}(1, \cdot), B_{\varepsilon}\right) \\
& =\operatorname{deg}\left(I-\mathcal{H}_{1}(0, \cdot), B_{\varepsilon}\right)=\operatorname{deg}\left(I, B_{\varepsilon}\right)=1 .
\end{aligned}
$$

Lemma 2.4. If $\lambda>\lambda_{1}$, then $i\left(\mathcal{K}_{\lambda}, 0\right)=0$.

Proof. Fix $\lambda>\lambda_{1}$ and $\phi \in X, \phi>0$. First, it is clear that there exists $\varepsilon>0$ such that

$$
\lambda-\varepsilon>\lambda_{1} .
$$


We define the map

$$
\mathcal{H}_{2}:[0,1] \times X \mapsto X ; \quad \mathcal{H}_{2}(t, u):=(-\Delta)^{-1}(f(u)+t \phi) .
$$

We will show that there exists $\delta>0$ such that $u \neq \mathcal{H}_{2}(t, u)$ for all $u \in \bar{B}_{\delta} \backslash\{0\}$ and $t \in[0,1]$. Indeed, otherwise there would exist sequences $u_{n} \in X \backslash\{0\}$ with $\left\|u_{n}\right\|_{\infty} \rightarrow 0$ and $t_{n} \in[0,1]$ such that $u_{n}=\mathcal{H}_{2}\left(t_{n}, u_{n}\right)$. Since $t_{n} \phi \geq 0$, we have that $u_{n}>0$ and so

$$
-\Delta u_{n}=u_{n}\left(\lambda+b \int_{\Omega} u_{n}^{r} d x-u_{n}\right)+t_{n} \phi>u_{n}(\lambda-\varepsilon)+t_{n} \phi \geq u_{n}(\lambda-\varepsilon),
$$

hence, $\lambda_{1} \geq \lambda-\varepsilon$, a contradiction with (2.3). This proves that the homotopy defined by $\mathcal{H}_{2}$ is admissible. Then, if we take $\varepsilon \in(0, \delta]$ we have

$$
i\left(\mathcal{K}_{\lambda}, 0\right)=\operatorname{deg}\left(\mathcal{K}_{\lambda}, B_{\varepsilon}\right)=\operatorname{deg}\left(I-\mathcal{H}_{2}(0, \cdot), B_{\varepsilon}\right)=\operatorname{deg}\left(I-\mathcal{H}_{2}(1, \cdot), B_{\varepsilon}\right)=0 .
$$

Proof of Theorem 2.2. The fact that $\lambda=\lambda_{1}$ is a bifurcation point follows from Lemmas 2.3 and 2.4. Moreover, if there exists a sequence $\left(\lambda_{n}, u_{n}\right)$ of positive solutions of (1.2) such that $\left\|u_{n}\right\|_{\infty} \rightarrow 0$, then, with a similar argument as in Lemma 2.3, we can easily conclude that $\lambda_{n} \rightarrow \lambda_{1}$. This proves that $\lambda_{1}$ is the only bifurcation point from the trivial solution. Hence, there exists an unbounded continuum of solutions of (1.2), see [13].

Now, we study the bifurcation direction. Assume that $b \leq 0$, then $-\Delta u \leq \lambda u$, that is, $\lambda \geq \lambda_{1}$.

Assume now that $b>0, r<1$ and that there exists a sequence $\left(\lambda_{n}, u_{n}\right)$ of positive solutions of (1.2) such that $\lambda_{n} \geq \lambda_{1}$ and $\left\|u_{n}\right\|_{\infty} \rightarrow 0$ as $n \rightarrow \infty$. Take $M>0$ such that

$$
1-b M \int_{\Omega} \varphi_{1} d x<0 .
$$

For $n$ large enough we have that $u_{n}^{r}>M u_{n}$, and then

$$
-\Delta u_{n} \geq u_{n}\left(\lambda_{n}+b M \int_{\Omega} u_{n} d x-u_{n}\right),
$$

so $u_{n}$ is a supersolution of

$$
-\Delta u=u\left(\lambda_{n}+b M \int_{\Omega} u_{n} d x-u\right) .
$$

Using Propositions 2.1 and (2.2), we get

$$
u_{n} \geq\left(\lambda_{n}+b M \int_{\Omega} u_{n} d x-\lambda_{1}\right) \varphi_{1}
$$

and hence

$$
\left(1-b M \int_{\Omega} \varphi_{1} d x\right) \int_{\Omega} u_{n} d x \geq\left(\lambda_{n}-\lambda_{1}\right) \int_{\Omega} \varphi_{1} d x
$$

a contradiction. 
Assume now that $b>0, r>1$ and that there exists a sequence $\left(\lambda_{n}, u_{n}\right)$ of positive solutions of (1.2) such that $\lambda_{n} \leq \lambda_{1}$ and $\left\|u_{n}\right\|_{\infty} \rightarrow 0$ as $n \rightarrow \infty$. Take $\varepsilon>0$ such that $1-b K \varepsilon|\Omega|>0$, where $K$ is defined in (2.2). For $n$ large enough we have $u_{n}^{r}<\varepsilon u_{n}$, and then

$$
-\Delta u_{n} \leq u_{n}\left(\lambda_{n}+b \varepsilon \int_{\Omega} u_{n} d x-u_{n}\right) .
$$

So, using again (2.2), we have

$$
u_{n} \leq\left(\lambda_{n}+b \varepsilon \int_{\Omega} u_{n} d x-\lambda_{1}\right) K,
$$

hence

$$
(1-b K \varepsilon|\Omega|) \int_{\Omega} u_{n} d x \leq\left(\lambda_{n}-\lambda_{1}\right) K|\Omega|,
$$

again a contradiction.

Finally, assume that $b>0$ and $r=1$. By the Crandall-Rabinowitz theorem (see [2]), there exist $\varepsilon>0$ and two regular functions $\lambda(s), u(s), s \in(-\varepsilon, \varepsilon)$, such that in a neighborhood of $\left(\lambda_{1}, 0\right)$ the unique positive solutions of (1.2) are $(\lambda(s), u(s)), s \in(0, \varepsilon)$. We can write

$$
u(s)=s \varphi_{1}+s^{2} \varphi_{2}+o\left(s^{2}\right), \quad \lambda(s)=\lambda_{1}+s \lambda_{2}+o(s),
$$

where $\lambda_{2} \in \mathbb{R}, \varphi_{2} \in C^{2}(\bar{\Omega})$. It is evident that the sign of $\lambda_{2}$ determines the bifurcation direction. Substituting these expansions into (1.2) and identifying the terms of order one in $s$ yields

$$
-\Delta \varphi_{2}-\lambda_{1} \varphi_{2}=\lambda_{2} \varphi_{1}-\varphi_{1}^{2}+b \varphi_{1} \int_{\Omega} \varphi_{1} d x
$$

Multiplying by $\varphi_{1}$, we conclude that

$$
\lambda_{2}=\frac{\int_{\Omega} \varphi_{1}^{3} d x-b \int_{\Omega} \varphi_{1}^{2} d x \int_{\Omega} \varphi_{1} d x}{\int_{\Omega} \varphi_{1}^{2} d x} .
$$

\section{A priori bounds and non-existence results of (1.2)}

In this section we obtain a priori bounds of solutions for $b>0$ as well as non-existence results of (1.2).

Proposition 3.1. Assume that $b>0, r<1$. Let $\left(\lambda, u_{\lambda}\right)$ be a positive solution of (1.2) such that $\lambda$ belongs to a compact set $\mathcal{K} \subset \mathbb{R}$. Then, $\left\|u_{\lambda}\right\|_{\infty} \leq C$ for a constant independent of $\lambda$. Moreover, if

$$
\lambda \leq \underline{\lambda}:=\left(\frac{1}{|\Omega| b r}\right)^{1 /(r-1)}\left(1-\frac{1}{r}\right),
$$

problem (1.2) does not possess any positive solution. 
Proof. Since $u_{\lambda}$ is a positive solution of (1.2) we have, using Proposition 2.1, that

$$
u_{\lambda} \leq \lambda+b \int_{\Omega} u_{\lambda}^{r} d x
$$

Using now that $\|u\|_{r} \leq|\Omega|^{(1-r) / r}\|u\|_{1}$, we have

$$
\int_{\Omega} u_{\lambda} d x-b|\Omega|^{2-r}\left(\int_{\Omega} u_{\lambda} d x\right)^{r} \leq \lambda|\Omega| \text {. }
$$

From (3.2) we get that if $\lambda \in \mathcal{K}$, then $\int_{\Omega} u_{\lambda} d x \leq C$, and so by (3.1) we get that $\left\|u_{\lambda}\right\|_{\infty} \leq C$, where by $C$ we denote different positive constants. On the other hand, the function

$$
f(s):=A s-B s^{q}, \quad A, B>0,0<q<1, s \geq 0,
$$

has a minimum at $s=s_{m}:=(A /(q B))^{1 /(q-1)}$ and

$$
f\left(s_{m}\right)=A^{q /(q-1)}\left(\frac{1}{B q}\right)^{1 /(q-1)}\left(1-\frac{1}{q}\right) .
$$

Hence, if

$$
\lambda|\Omega| \leq\left(\frac{1}{b|\Omega|^{2-r} r}\right)^{1 /(r-1)}\left(1-\frac{1}{r}\right)
$$

then by (3.2), equation (1.2) does not have positive solutions.

Proposition 3.2. Assume that $b>0, r>1$. Let $\left(\lambda, u_{\lambda}\right)$ be a positive solution of (1.2) such that $\lambda$ belongs to a compact set $\mathcal{K} \subset \mathbb{R}$. Then, $\left\|u_{\lambda}\right\|_{\infty} \leq C$ for a constant independent of $\lambda$. Moreover, if

$$
\lambda \geq \bar{\lambda}:=\lambda_{1}+b^{1 /(1-r)}\left(\int_{\Omega} \varphi_{1} d x\right)^{r /(1-r)}|\Omega| r^{r /(1-r)}(r-1),
$$

problem (1.2) does not possess any positive solution.

Proof. Using now the lower bound in Proposition 2.1, we get that

$$
\left(\lambda-\lambda_{1}+b \int_{\Omega} u_{\lambda}^{r} d x\right) \varphi_{1} \leq u_{\lambda}
$$

and then

$$
\left(\lambda-\lambda_{1}+b \int_{\Omega} u_{\lambda}^{r} d x\right) \int_{\Omega} \varphi_{1} d x \leq \int_{\Omega} u_{\lambda} d x \leq|\Omega|^{(r-1) / r}\left(\int_{\Omega} u_{\lambda}^{r} d x\right)^{1 / r},
$$

hence

$$
b \int_{\Omega} \varphi_{1} d x \int_{\Omega} u_{\lambda}^{r} d x-|\Omega|^{(r-1) / r}\left(\int_{\Omega} u_{\lambda}^{r} d x\right)^{1 / r} \leq\left(\lambda_{1}-\lambda\right) \int_{\Omega} \varphi_{1} d x .
$$

From (3.4) we get that if $\lambda \in \mathcal{K}$, then $\int_{\Omega} u_{\lambda}^{r} \leq C$, and hence by (3.1), $\left\|u_{\lambda}\right\|_{\infty} \leq C$. On the other hand, applying again the results of (3.3) with

$$
A=b \int_{\Omega} \varphi_{1}, \quad B=|\Omega|^{(r-1) / r}, \quad q=1 / r
$$


we get that if

$$
\left(\lambda_{1}-\lambda\right) \int_{\Omega} \varphi_{1} d x \leq b^{1 /(1-r)}\left(\int_{\Omega} \varphi_{1} d x\right)^{1 /(1-r)}|\Omega| r^{r /(1-r)}(1-r)
$$

then by (3.4), equation (1.2) does not have positive solutions.

For the case $r=1$, the bounds depend on the size of $b$.

Proposition 3.3. Assume that $b>0, r=1$. Assume that $b<1 /|\Omega|$ or $b \int_{\Omega} \varphi_{1} d x>1$, then there exist a priori bounds of the solution of (1.2). Moreover, if $b<1 /|\Omega|$ and $\lambda \leq 0$ or $b \int_{\Omega} \varphi_{1} d x>1$ and $\lambda \geq \lambda_{1}$, then (1.2) does not possess any positive solution.

Proof. In this case, by $(2.2)$ we get

$$
\left(\lambda+b \int_{\Omega} u d x-\lambda_{1}\right) \varphi_{1} \leq u \leq \lambda+b \int_{\Omega} u d x
$$

and so

(3.6) $(1-b|\Omega|) \int_{\Omega} u d x \leq \lambda|\Omega|, \quad\left(b \int_{\Omega} \varphi_{1} d x-1\right) \int_{\Omega} u d x \leq\left(\lambda_{1}-\lambda\right) \int_{\Omega} \varphi_{1} d x$.

From these inequalities we obtain the result.

\section{Stability and uniqueness results}

In this section we study the stability of a positive solution $u$ of (1.2) when $b>0$. In order to ascertain its stability we have to calculate the sign of the principal eigenvalue of the linearized problem around $u$, that is,

$$
\begin{cases}-\Delta \xi+\left(2 u-\lambda-b \int_{\Omega} u^{r} d x\right) \xi-b r u \int_{\Omega} u^{r-1} \xi d x=\sigma \xi & \text { in } \Omega, \\ \xi=0 & \text { on } \partial \Omega .\end{cases}
$$

This problem is a non-local and singular (when $r<1$ ) eigenvalue problem which has been analyzed in other papers (see [4] and Section 5 in [12]) and it is included in the general problem

$$
\begin{cases}-\Delta \xi+m(x) \xi-a_{1}(x) \int_{\Omega} a_{2}(x) \xi d x=\sigma \xi & \text { in } \Omega \\ \xi=0 & \text { on } \partial \Omega\end{cases}
$$

where $m, a_{1} \in C^{1}(\bar{\Omega}), a_{2} \in C(\Omega), a_{1}, a_{2}>0$ and $a_{2}(x) \leq K d(x, \partial \Omega)^{-\beta}, \beta<1$, $K>0$. The existence of a principal eigenvalue of $(4.2)$, denoted by $\lambda_{1}(-\Delta+$ $\left.m ; a_{1} ; a_{2}\right)$, has been established. If no confusion arises, we write $\lambda_{1}(-\Delta+m)$ when $a_{1}$ or $a_{2}$ vanishes (observe that $\lambda_{1}(-\Delta+m)$ is the classical principal eigenvalue of a local eigenvalue problem).

In the following result we give criteria for the sign of $\lambda_{1}\left(-\Delta+m ; a_{1} ; a_{2}\right)$. The proof, an adaptation of the characterization theorem of the maximum principle established in Theorem 7.10 of [16], can be found in [4]. 
Proposition 4.1.

(a) Assume that there exists a positive function $\bar{u} \in C^{2}(\Omega) \cap C_{0}^{1, \delta}(\bar{\Omega}), \delta \in$ $(0,1)$, such that

$$
-\Delta \bar{u}+m(x) \bar{u}-a_{1}(x) \int_{\Omega} a_{2}(x) \bar{u} d x>0 \quad \text { in } \Omega,
$$

(we say that $\bar{u}$ is a supersolution of (4.2)). Then, $\lambda_{1}\left(-\Delta+m ; a_{1} ; a_{2}\right)>0$.

(b) Assume that there exists a positive function $\underline{u} \in C^{2}(\Omega) \cap C_{0}^{1, \delta}(\bar{\Omega}), \delta \in$ $(0,1)$, such that

$$
-\Delta \underline{u}+m(x) \underline{u}-a_{1}(x) \int_{\Omega} a_{2}(x) \underline{u} d x<0 \quad \text { in } \Omega .
$$

(we say that $\underline{u}$ is a subsolution of $(4.2))$. Then, $\lambda_{1}\left(-\Delta+m ; a_{1} ; a_{2}\right)<0$.

In the following result, we study the sign of the principal eigenvalue in some specific cases.

Proposition 4.2. Assume that $b>0$.

(a) Assume $r \leq 1$ and $\lambda>\lambda_{1}$. There exists $b_{1}>0$ such that for $0<b<b_{1}$, any positive solution is stable.

(b) Assume $r \geq 1$ and $\lambda \leq 0$. Then, any positive solution is unstable.

Proof. (a) Observe that in our case

$$
m(x)=2 u_{b}-\lambda-b \int_{\Omega} u_{b}^{r} d x, \quad a_{1}=b r u_{b}, \quad a_{2}=u_{b}^{r-1},
$$

where $u_{b}$ is a positive solution of (1.2). By the strong maximum principle, $u_{b}$ is strongly positive. Hence, there exist $0<k_{1}<k_{2}$ such that $k_{1} d(x, \partial \Omega) \leq$ $u_{b} \leq k_{2} d(x, \partial \Omega)$, and then $a_{2}$ verifies the hypothesis $a_{2}(x) \leq K d(x, \partial \Omega)^{-\beta}$ for $\beta=1-r$.

On the other hand, since $u_{b}$ is a positive solution of (1.2), then

$$
\lambda_{1}\left(-\Delta+u_{b}-\lambda-b \int_{\Omega} u_{b}^{r} d x\right)=0
$$

and so, by the monotonicity of the principal eigenvalue with respect to the zero order term, $\lambda_{1}\left(-\Delta+2 u_{b}-\lambda-b \int_{\Omega} u_{b}^{r} d x\right)>0$. Consider $e_{b}>0$ the unique positive solution of the linear equation

$$
\begin{cases}-\Delta e_{b}+\left(2 u_{b}-\lambda-b \int_{\Omega} u_{b}^{r} d x\right) e_{b}=r u_{b} & \text { in } \Omega \\ e_{b}=0 & \text { on } \partial \Omega .\end{cases}
$$

Now, we apply Proposition 4.1. It is clear that $e_{b}$ is a supersolution of (4.1) if

$$
\frac{1}{b}>\int_{\Omega} u_{b}^{r-1} e_{b} d x
$$


We claim that

$$
u_{b} \rightarrow \theta_{\lambda} \quad \text { in } C^{2}(\bar{\Omega}) \text { as } b \rightarrow 0 .
$$

Observe that (4.5) implies that $e_{b} \rightarrow e_{\lambda}$ in $C^{2}(\bar{\Omega})$ as $b \rightarrow 0$, where $e_{\lambda}$ is the unique positive solution of

$$
-\Delta e_{\lambda}+\left(2 \theta_{\lambda}-\lambda\right) e_{\lambda}=r \theta_{\lambda} \quad \text { in } \Omega, \quad e_{\lambda}=0 \quad \text { on } \partial \Omega .
$$

Hence, we conclude that (4.4) holds for small $b$, then $u_{b}$ is stable.

We prove (4.5). Assume $r<1$, then using (3.2) and (3.1) we get that

$$
\left\|u_{b}\right\|_{\infty} \leq C(\lambda, b)
$$

where $C$ is a constant bounded when $b \rightarrow 0$. Hence,

$$
b \int_{\Omega} u_{b}^{r} d x \rightarrow 0 \quad \text { as } b \rightarrow 0
$$

and we conclude with (4.5). Assume now that $r=1$, in this case by (3.5) and (3.6), we conclude that

$$
\left\|u_{b}\right\|_{\infty} \leq \frac{\lambda}{1-b|\Omega|}
$$

We can repeat the above reasoning to conclude with (4.5).

(b) In a similar way, $u_{b}$ is a subsolution of (4.1) provided that

$$
u_{b} \leq b r \int_{\Omega} u_{b}^{r} d x
$$

Since $u_{b} \leq \lambda+b \int_{\Omega} u_{b}^{r} d x$, it follows that (4.8) holds for $\lambda \leq 0$ and $r \geq 1$.

Corollary 4.3. Assume $0<b<b_{1}, r \leq 1$ and $\lambda>\lambda_{1}$, where $b_{1}$ is from Proposition 4.2. Then, there exists a unique positive solution of (1.2).

Proof. We use the fixed point index in cones. Define $P:=\{u \in X: u \geq$ 0 in $\Omega\}$. Assume that $r \leq 1$ and $b<b_{1}$, then using (4.6) and (4.7) there exists $R_{1}$ independent of $b$ such that $\|u\|_{L^{\infty}(\Omega)} \leq R_{1}$, for all positive solutions $u$ of (1.2).

Finally, take $M>0$ large enough and consider the operator $\mathcal{K}: X \mapsto X$ defined by

$$
\mathcal{K}(u):=(-\Delta+M)^{-1}\left(u\left(\lambda+M-u+b \int_{\Omega} u^{r} d x\right)\right) .
$$

It is clear that $\mathcal{K}$ is a positive operator whose fixed points are non-negative solutions of (1.2). Hence, the fixed point index of $\mathcal{K}$ over $\mathcal{B}$ with respect to the cone $P$ is well-defined, where $\mathcal{B}:=\left\{u \in P:\|u\|_{L^{\infty}(\Omega)} \leq R_{1}+1\right\}$.

Now, we are going to compute this index in particular cases. We claim that, if $\lambda>\lambda_{1}$, then

(I.1) $i_{P}(\mathcal{K}, \mathcal{B})=1$, 
(I.2) $i_{P}(\mathcal{K}, 0)=0$

(I.3) $i_{P}\left(\mathcal{K}, u_{b}\right)=1$,

for any positive solution $u_{b}$ of (1.2). Of course, we conclude the uniqueness of positive solution of (1.2).

(I.2) follows by a similar argument as the one used in the proof of Lemma 2.4. Proposition 4.2 implies (I.3). Finally, we show (I.1). Consider the operator $\mathcal{H}_{1}:[0,1] \times X \mapsto X$ defined by

$$
\mathcal{H}_{1}(t, u):=(-\Delta+M)^{-1}\left(u\left(\lambda+M-u+t b \int_{\Omega} u^{r} d x\right)\right) .
$$

By the a priori bounds, $\mathcal{H}_{1}$ has no fixed points on $\partial \mathcal{B}$ for $t \in[0,1]$. Thus, it follows by homotopy invariance that

$$
i_{P}(\mathcal{K}, \mathcal{B})=i_{P}\left(\mathcal{H}_{1}(1, \cdot), \mathcal{B}\right)=i_{P}\left(\mathcal{H}_{1}(0, \cdot), \mathcal{B}\right)=1
$$

This last inequality follows because $u=\mathcal{H}_{1}(0, u)$ is equivalent to the classical equation (2.1), and for this equation it is well-known that the fixed point index is equal to one.

\section{Proofs of Theorems 1.1-1.4}

Proof of Theorem 1.1. By Theorem 2.2, we know the existence of an unbounded continuum of positive solutions bifurcating from the trivial solution at $\lambda=\lambda_{1}$. Since $-\Delta u \leq u(\lambda-u)$, we know that positive solutions do not exist for $\lambda \leq \lambda_{1}$ and that for any solution $u \leq \theta_{\lambda}$. Hence we conclude the existence of positive solution for $\lambda>\lambda_{1}$.

We show now the uniqueness. Assume that there exist two positive solutions $u \neq v$. If $\int_{\Omega} u^{r} d x=\int_{\Omega} v^{r} d x$ then we conclude easily that $u=v$. So, assume that for instance $\int_{\Omega} u^{r} d x>\int_{\Omega} v^{r} d x$. Then,

$$
-\Delta u=u\left(\lambda+b \int_{\Omega} u^{r} d x-u\right)<u\left(\lambda+b \int_{\Omega} v^{r} d x-u\right),
$$

and then by Proposition 2.1 we get $u<v$, a contradiction.

On the other hand, we have

$$
u \leq \lambda+b \int_{\Omega} u^{r} d x
$$

then $u \leq \lambda$. So, as $b \rightarrow-\infty$ we get

$$
\int_{\Omega} u^{r} d x \rightarrow 0
$$

Moreover, as

$$
\left(\lambda+b \int_{\Omega} u^{r} d x-\lambda_{1}\right) \varphi_{1} \leq u \quad \text { and } \quad \lambda+b \int_{\Omega} u^{r} d x-\lambda_{1}>0
$$

we conclude that $b \int_{\Omega} u^{r} d x \rightarrow \lambda_{1}-\lambda$. This implies that $\|u\|_{\infty} \rightarrow 0$. 
Proof of Theorem 1.2. Assume $b>0$ and $r<1$. Define

$\lambda_{*}:=\inf \{\lambda \in \mathbb{R}:(1.2)$ possesses at least one positive solution $\}$.

We know by Theorem 2.2 and Proposition 3.1 that $-\infty<\lambda_{*}<\lambda_{1}$. We prove now that there exists a positive solution for all $\lambda>\lambda_{*}$, for which we are going to use the sub-supersolution method, see for instance [3]. Indeed, take $\lambda>\lambda_{*}$, then there exists $\mu \in\left[\lambda_{*}, \lambda\right)$ such that (1.2) possesses at least one positive solution, denote it by $u_{\mu}$. Now, it is clear that $(\underline{u}, \bar{u})=\left(u_{\mu}, K\right)$ is a sub-supersolution of (1.2) for $K$ large enough, specifically for $K$ verifying $K-b K^{r}|\Omega| \geq \lambda$. Enlarging if necessary $K$ so that $u_{\mu} \leq K$ we conclude the existence of a positive solution for $\lambda$.

Finally, take a sequence of positive solutions $\left(\lambda_{n}, u_{n}\right)$ of (1.2) such that $\lambda_{n} \geq$ $\lambda_{*}$ and $\lambda_{n} \rightarrow \lambda_{*}$. Thanks to the bounds of Proposition 3.1 we have that $u_{n} \rightarrow$ $u_{*} \geq 0, u_{*}$ is a solution for $\lambda=\lambda_{*}$. Since $\lambda_{*}<\lambda_{1}$ and $\lambda_{1}$ is the unique bifurcation point from the trivial solution, we conclude that $u_{*}>0$.

On the other hand, since $u$ is bounded and

$$
\lambda+b \int_{\Omega} u^{r} d x>\lambda_{1}
$$

and then taking $b \rightarrow 0$ we have that $\lambda \geq \lambda_{1}$, that is $\lim _{b \rightarrow 0} \lambda_{*}(b)=\lambda_{1}$.

Finally, we prove that $\lim _{b \rightarrow \infty} \lambda_{*}(b)=-\infty$, for that it suffices to show that for any $\lambda<\lambda_{1}$ there exists $b>0$ large enough such that (1.2) possesses at least one positive solution. Fix $\lambda<\lambda_{1}$, there exists $b>0$ large enough (see (3.3)) such that the function $f(s)=s-s^{r} b \int_{\Omega} \varphi_{1}^{r} d x$ has a minimum $s_{m}$ such that $f\left(s_{m}\right)<\lambda-\lambda_{1}$. Fixing such $b$, take $\varepsilon>0$ such that $f(\varepsilon)<\lambda-\lambda_{1}$. Then, $(\underline{u}, \bar{u})=\left(\varepsilon \varphi_{1}, K\right)$ is sub-supersolution of (1.2) for $K$ large enough. Indeed, $\underline{u}$ is a subsolution if

$$
\varepsilon \varphi_{1}-b \varepsilon^{r} \int_{\Omega} \varphi_{1}^{r} d x \leq \lambda-\lambda_{1}
$$

that is, taking into account that $\left\|\varphi_{1}\right\|_{\infty}=1, f(\varepsilon)<\lambda-\lambda_{1}$.

Proof of Theorem 1.3. Assume that $b>0$ and $r>1$. Define now

$$
\lambda^{*}:=\sup \{\lambda \in \mathbb{R}:(1.2) \text { possesses at least one positive solution }\} .
$$

We know by Theorem 2.2 and Proposition 3.2 that $\lambda_{1}<\lambda^{*}<+\infty$. We prove now that there exists a positive solution for all $\lambda \in\left[\lambda_{1}, \lambda^{*}\right)$ and observe that for $\lambda \leq \lambda_{1}$ positive solutions exist. Indeed, take $\lambda<\lambda^{*}$, then there exists $\mu \in\left(\lambda, \lambda^{*}\right]$ such that (1.2) possesses at least one positive solution, denote it by $u_{\mu}$. Now, it is clear that $(\underline{u}, \bar{u})=\left(\varepsilon \varphi_{1}, u_{\mu}\right)$ is a sub-supersolution of (1.2) for $\varepsilon>0$ small enough, specifically for $\varepsilon$ verifying

$$
\varepsilon-b \varepsilon^{r} \int_{\Omega} \varphi_{1}^{r} d x \leq \lambda-\lambda_{1} \quad \text { and } \quad \varepsilon \varphi_{1} \leq u_{\mu} .
$$


Finally, taking a sequence of solutions $\left(\lambda_{n}, u_{n}\right)$ with $\lambda_{n} \leq \lambda^{*}, \lambda_{n} \rightarrow \lambda^{*}$ and thanks to the bounds of Proposition 3.2, we have that $u_{n} \rightarrow u^{*}>0$, where $u^{*}$ is the unique positive solution for $\lambda=\lambda^{*}$. Observe that since $\lambda_{1}<\lambda^{*} \leq \bar{\lambda}$, where $\bar{\lambda}$ is defined in Proposition 3.2 and $\lim _{b \rightarrow \infty} \bar{\lambda}(b)=\lambda_{1}$ we conclude that $\lim _{b \rightarrow \infty} \lambda^{*}(b)=\lambda_{1}$.

Finally, we prove that $\lim _{b \rightarrow 0} \lambda^{*}(b)=+\infty$, for that it suffices to show that for any $\lambda>\lambda_{1}$, there exists $b>0$ small enough such that (1.2) possesses at least one positive solution. Let us fix $\lambda>\lambda_{1}$, take $\widetilde{\Omega} \supset \Omega$ and consider $\widetilde{\varphi}_{1}$ and $\widetilde{\lambda}_{1}$ the positive eigenfunction and eigenvalue associated to $\widetilde{\Omega}$. Consider the function

$$
g(s):=s\left(\widetilde{\varphi}_{1}\right)_{L}-b s^{r} \int_{\Omega} \varphi_{1}^{r} d x
$$

where $\left(\widetilde{\varphi}_{1}\right)_{L}:=\min _{x \in \bar{\Omega}} \varphi_{1}(x)$. This function attains its maximum at

$$
s=s_{M}=\left(\left(\widetilde{\varphi}_{1}\right)_{L} /\left(b \int_{\Omega} \varphi_{1}^{r} d x\right)\right)^{1 /(r-1)}
$$

and

$$
g\left(s_{M}\right)=\left(\widetilde{\varphi}_{1}\right)_{L}^{r /(r-1)}\left(1 /\left(b \int_{\Omega} \varphi_{1}^{r} d x\right)\right)^{1 /(r-1)}\left(1-\frac{1}{r}\right) .
$$

Hence for $b$ small enough we get $g\left(s_{M}\right)>\lambda-\widetilde{\lambda}_{1}$. Take $K>0$ such that $g(K)>\lambda-\widetilde{\lambda}_{1}$. Fixing such $b$ and $K$, we have that $(\underline{u}, \bar{u})=\left(\varepsilon \varphi_{1}, K \widetilde{\varphi_{1}}\right)$ is a sub-supersolution of (1.2) for small $\varepsilon$.

Proof of Theorem 1.4. By Theorem 2.2, there exists an unbounded continuum of positive solutions bifurcating from the trivial solution at $\lambda=\lambda_{1}$. Assume $b<1 /|\Omega|$, then by Proposition 3.3 there does not exist any positive solution for $\lambda \leq 0$ and the positive solutions are bounded. Hence the existence of positive solution for $\lambda>\lambda_{1}$ is obtained.

Assume now that $b>1 / \int_{\Omega} \varphi_{1} d x$. In this case by Proposition 3.3 we know that for $\lambda \geq \lambda_{1}$ there does not exist any positive solution and that the positive solutions are bounded for $\lambda \leq \lambda_{1}$. Hence the existence of positive solution for $\lambda<\lambda_{1}$ is obtained.

\section{The parabolic problem}

Consider now the time dependent problem (1.1). The existence and uniqueness of the local positive solution follows from the classical theory, see for instance Example 51.13 in [19]. Moreover, the solution can be extended in time if the $L^{\infty}$-norm remains finite. First, we show that in the case $b<0$ the solution is global and bounded.

Lemma 6.1. Assume $b \leq 0$. Then, the positive solution $u$ of (1.1) is global in time and bounded. Moreover, if $\lambda<\lambda_{1}$ we get $\|u(x, t)\|_{\infty} \rightarrow 0$ as $t \rightarrow \infty$. 
Proof. If $b<0$, the solution $u$ of (1.1) is a subsolution of the local logistic equation

$$
U_{t}-\Delta U=U(\lambda-U), \quad U(x, 0)=u_{0}(x) .
$$

It is well-known, see for instance [20], that the above equation is global and bounded and that $u \leq U$. Finally, $\|U(x, t)\|_{\infty} \rightarrow 0$ as $t \rightarrow \infty$ for $\lambda<\lambda_{1}$ and this completes the proof.

Now, we consider the case $b>0$. In this case, thanks to the maximum principle (see again [20] or [22]) we can assume that $u_{0}(x)>0$ for $x \in \Omega$ and $u_{0}(x)=0$ on $\partial \Omega$.

TheOREM 6.2 (Global existence results). Assume $b>0$.

(a) If $r<1$, the solution exists globally in time for all $\lambda \in \mathbb{R}$.

(b) If $r=1$ and $b|\Omega|<1$, the solution exists globally in time for all $\lambda \in \mathbb{R}$.

(c) If $r=1$ and $b|\Omega| \geq 1$, the solution exists globally in time for all $\lambda<0$ if

$$
u_{0}(x) \leq \frac{\lambda}{1-b|\Omega|} \quad \text { for all } x \in \Omega .
$$

(d) Assume $r=1$. Let $e$ be the unique positive solution of

$$
\begin{cases}-\Delta e=1 & \text { in } \Omega \\ e=0 & \text { on } \partial \Omega .\end{cases}
$$

Then, there exists a small number $a_{1}>0$ such that, if $u_{0}(x) \leq a_{1} e(x)$ for $x \in \Omega$, the solution exists globally in time for all $\lambda \in\left(-\infty, 1 / \max _{x \in \bar{\Omega}} e(x)\right)$.

(e) Assume $r>1$, then, there exists $a_{2}>0$ (which can be computed explicitly) such that the solution exists globally in time, for all

$$
\lambda \in\left(-\infty,\left(\frac{1}{b|\Omega| r}\right)^{1 /(r-1)} \frac{r-1}{r}\right)
$$

provided that $u_{0}(x) \leq a_{2}$.

Proof. For the first three paragraphs, use $\bar{u}(x, t)=M$ as a supersolution, where $M$ is a positive constant. Indeed, $\bar{u}$ is a supersolution of (1.1) if

$$
M \geq \lambda+b|\Omega| M^{r}, \quad M \geq u_{0}(x) .
$$

For (e), observe that the function $g(M)=\lambda+b|\Omega| M^{r}-M$ goes to $+\infty$ as $M \rightarrow+\infty$ if $r>1$ and attains a minimum at $M_{m}=(1 /(b|\Omega| r))^{1 /(r-1)}$. It is enough to impose that $g\left(M_{m}\right) \leq 0$ and $a_{2}$ will be defined by $g\left(a_{2}\right)=0$. Finally, for $(\mathrm{d})$, take $\bar{u}(x, t)=a_{1} e(x)$. It is clear that $\bar{u}$ is supersolution if

$$
1>\lambda e+a_{1} e\left(b \int_{\Omega} e(x) d x-e\right), \quad a_{1} e(x) \geq u_{0}(x) .
$$

If $1>\lambda e$ we can take $a_{1}$ small enough. 
The next result studies the case when the solution goes to zero:

Proposition 6.3. Assume $b>0$.

(a) If $r>0$, the trivial solution is locally exponentially stable if $\lambda<\lambda_{1}$.

(b) If $r<1$, there exists $\underline{\lambda}$ such that for all $\lambda<\underline{\lambda}$ and initial datum $u_{0}$, we have $\|u(x, t)\|_{\infty} \rightarrow 0$ as $t \rightarrow \infty$.

(c) If $r=1$ and $b$ small enough, then for $\lambda<\lambda_{1}$ and for all initial datum $u_{0}$, we have $\|u(x, t)\|_{\infty} \rightarrow 0$ as $t \rightarrow \infty$.

(d) If $r>1$, then for all $u_{0}$ there exists $\underline{\lambda}\left(u_{0}\right)$ such that for $\lambda<\underline{\lambda}\left(u_{0}\right)$, we have $\|u(x, t)\|_{\infty} \rightarrow 0$ as $t \rightarrow \infty$.

PRoOF. First, take a domain $\Omega_{1} \supset \Omega$ such that, if necessary,

$$
\lambda<\mu_{1}<\lambda_{1}
$$

where $\mu_{1}$ is the principal eigenvalue associated to $-\Delta$ in $\Omega_{1}$ and denote by $\psi_{1}$ the positive eigenfunction associated to $\mu_{1}$ such that $\left\|\psi_{1}\right\|_{\infty}=1$.

In all cases, we take $\bar{u}(x, t)=M e^{-\sigma t} \psi_{1}$ as a supersolution, with $M>0$ and $\sigma>0$ to be chosen. It is clear that $\bar{u}$ is a supersolution of (1.1) if

(6.3) $M \psi_{1}(x) \geq u_{0}(x), \quad x \in \Omega \quad$ and $\quad-\sigma+\mu_{1}-\lambda \geq-M e^{-\sigma t} R+b M^{r} e^{-r \sigma t} B$, where $R:=\min _{x \in \bar{\Omega}} \psi_{1}(x)$ and $B:=\int_{\Omega} \psi_{1}^{r} d x$.

For the first paragraph, take $M$ small enough, then it suffices to take $0<$ $\sigma<\mu_{1}-\lambda$ what is possible thanks to (6.2). For the second one $(r<1)$, observe that

$$
-M e^{-\sigma t} R+b M^{r} e^{-r \sigma t} B \leq C
$$

for some positive constant $C$ independent of $t$ and $M$. It suffices to take $\lambda$ negative. When $r=1$, then

$$
-M e^{-\sigma t} R+b M e^{-\sigma t} B=M e^{-\sigma t}(-R+b B)<0
$$

for $b$ small enough. Fixing this value of $b$, take $\lambda<\lambda_{1}$ and $\sigma>0$.

For the last paragraph $(r>1)$, for a given $u_{0}$ take $M$ such that $u_{0} \leq M \psi_{1}$. Fixed such $M$, take $\lambda$ small enough such that (6.3) is verified.

Theorem 6.4 (Blow-up in finite time). Assume $b>0$.

(a) Assume $r=1$ and define

$$
A:=\int_{\Omega} \varphi_{1} d x
$$

If $b A=1$ and $\lambda>\lambda_{1}$ the solution $\|u(x, t)\|_{\infty}$ goes to $\infty$ as $t \rightarrow \infty$. In the case $b A>1$ the solution blows up in finite time for $\lambda>\lambda_{1}$ or for any $\lambda$ if $u_{0}$ is large enough.

(b) Assume $r>1$. Then, there exists $\bar{\lambda}$ such that for $\lambda>\bar{\lambda}$ the solution blows up in finite time for any $u_{0}$. 
(c) Assume $r>1$. Then, there exists $b_{2}>0$ such that the solution blows up in finite time if $u_{0}(x) \geq b_{2} \varphi_{1}(x)$.

Proof. (a) Take $\underline{u}(x, t)=q(t) \varphi_{1}(x)$ with $q(t)$ and $q(0)>0$ to be chosen. Observe that $\underline{u}$ is a subsolution of (1.1) if

$$
q^{\prime}(t) \leq\left(\lambda-\lambda_{1}\right) q-q^{2} \varphi_{1}+b q^{r+1} B \quad \text { and } \quad q(0) \varphi_{1}(x) \leq u_{0}(x)
$$

with $B:=\int_{\Omega} \varphi_{1}^{r} d x$. Since $\left\|\varphi_{1}\right\|_{\infty}=1$, we can take $q$ such that

$$
q^{\prime}(t)=\left(\lambda-\lambda_{1}\right) q-q^{2}+b q^{r+1} B .
$$

If $r=1$ the results follow easily. Indeed, in this case the above equation can be written as

$$
q^{\prime}(t)=\left(\lambda-\lambda_{1}\right) q+q^{2}(-1+b A) .
$$

(b) Assume that $r>1$. It can be proved that for $1<p<r+1$, there exists $\mu \in \mathbb{R}$ such that

$$
\left(\lambda-\lambda_{1}\right) q-q^{2}+b q^{r+1} B \geq \mu q+q^{p} .
$$

Indeed, this is equivalent to $\lambda-\lambda_{1}-\mu \geq q-b B q^{r}+q^{p-1}$, and observe that the function $h(q)=q-b q^{r}+q^{p-1}$ is bounded. Taking $\mu=0$, the above inequality for $\lambda$ large enough, and hence $q^{\prime} \geq q^{p}$ and so $q$ blows up in finite time.

(c) We take $\mu<0$ with $|\mu|$ large enough, and hence in this case $q^{\prime} \geq \mu q+q^{p}$. In this case, $q$ blows up in finite time for $q(0)>0$ large enough, that is, for $u_{0}$ large enough.

REMARK 6.5. (a) Remember that for $r \leq 1$ and $b$ small enough the steadystate problem (1.2) has a unique positive solution. Then, using arguments of [20] (see for instance Theorem 5.4.4) the solution of (1.1) converges to the unique positive solution of (1.2).

(b) The blow-up in finite time of problem (1.3) has been studied in [15]. In order to compare the results of [15] with ours, let us assume that $r=1$ and fix the function $a$. In [15] it was proved that that there exists a value $\lambda^{*}>0$ (related with some eigenvalue problem associated to $a^{+}$) such that:

- If $\lambda_{1}<\lambda<\lambda^{*}$, then the solution of (1.3) blows up if

$$
b>A\left(\lambda, u_{0}\right)
$$

for some specific positive constant $A$ depending on $\lambda$ and $u_{0}$. Moreover, the maps $\lambda \mapsto A\left(\lambda, u_{0}\right)$ and $u_{0} \mapsto A\left(\lambda, u_{0}\right)$ are decreasing.

- If $\lambda \geq \lambda^{*}$, the solution of (1.3) blows up for any $u_{0}$.

Hence, as a consequence, the solution of (1.3) blows up for any $b$ if $\lambda$ is large enough or any $\lambda$ and $u_{0}$ large enough. However, in our results we need to require that $b$ is large enough to obtain that the solution of (1.1) blows up. 
Acknowledgements. We would like to thank the referee for a careful reading and comments on the work.

\section{REFERENCES}

[1] W. Allegretto and A. Barabanova, Existence of positive solutions of semilinear elliptic equations with nonlocal terms, Funkcial. Ekvac. 40 (1997), 395-409.

[2] M. G. Crandall and P.H. Rabinowitz, Bifurcation from simple eigenvalues, J. Funct. Anal. 8 (1971), 321-340.

[3] F.J.S.A. Corrêa, M. Delgado and A. SuÁrez, Some nonlinear heterogeneous problems with nonlocal reaction term, Adv. Differential Equations 16 (2011), 623-641.

[4] _ Some non-local population models with non-linear diffusion, Math. Comput. Modelling 54 (2011), 2293-2305.

[5] F.A. Davidson And N. Dodds, Existence of positive solutions due to nonlocal interactions in a class of nonlinear boundary value problems, Methods Appl. Anal. 14 (2007), 15-27.

[6] M. Delgado, J. López-Gómez and A. SuÁrez, On the symbiotic Lotka-Volterra model with diffusion and transport effects, J. Differential Equations 160 (2000), 175-262.

[7] P. Freitas, Nonlocal reaction-diffusion equations, Differential equations with applications to biology (Halifax, NS, 1997), 187-204, Fields Inst. Commun., 21, Amer. Math. Soc., Providence, RI, 1999.

[8] J. Furter and M. Grinfeld, Local vs. nonlocal interactions in population dynamics, J. Math. Biol. 27 (1989), 65-80.

[9] J. García-Melián, Multiplicity of positive solutions to boundary blow-up elliptic problems with signchanging weights, J. Funct. Anal. 261 (2011), 1775-1798.

[10] B. Gidas AND J. Spruck, A priori bounds for positive solutions of nonlinear elliptic equations, Comm. Partial Differential Equations 6 (1981), 883-901.

[11] S. B. Hsu, J. López-Gómez, L. Mei and M. Molina-Meyer, A nonlocal problem from conservation biology, SIAM J. Math. Anal. 46 (2014), 435-459.

[12] J. LÓPEZ-GómEZ, On the structure and stability of the set of solutions of a nonlocal problem modeling Ohmic heating, J. Dynam. Differential Equations 10 (1998), 537-566.

[13] _ Spectral Theory and Nonlinear Functional Analysis, Research Notes in Mathematical Series 426, Chapman \& Hall / CRC, Florida 2001.

[14] _ Varying bifurcation diagrams of positive solutions for a class of indefinite superlinear boundary value problems, Trans. Amer. Math. Soc. 352 (2000), 1825-1858.

[15] _ Global existence versus blow-up in superlinear indefinite parabolic problems, Sci. Math. Jpn. 61 (2005), 493-516.

[16] L Linear Second Order Elliptic Operators, World Scientific Publishing Co. Pte. Ltd., Hackensack, NJ, 2013.

[17] J. López-Gómez And P. Quittner, Complete and energy blow-up in indefinite superlinear parabolic problems, Crete Contin. Dyn. Syst. 14 (2006), 169-186.

[18] J. López-Gómez, A. Tellini and F. Zanolin, High multiplicity and complexity of the bifurcation diagrams of large solutions for a class of superlinear indefinite problems, Commun. Pure Appl. Anal. 13 (2014), 1-73.

[19] P. Quittner, And P. Souplet, Superlinear Parabolic Problems. Blow-up, Global Existence and steady States, Birkhäuser Advanced Texts: Basel Textbooks, Verlag, Basel, 2007.

[20] _ Nonlinear Parabolic and Elliptic Equations, Plenum Press, New York, 1992.

[21] P. Rouchon, Boundedness of global solutions of nonlinear diffusion equation with localized reaction term, Differential Integral Equations 16 (2003), 1083-1092. 
[22] M. WAng, AND Y. Wang, Properties of positive solutions for non-local reaction-diffusion problems, Math. Meth. Appl. Scienc. 19 (1996), 1141-1156.

[23] Y. Yamada, On Logistic Diffusion Equations with Nonlocal Effects, Proceedings of Seminar on Partial Differential Equations in Osaka 2012 in honor of Professor Hiroki Tanabe's 80th birthday (Osaka University, August 20-24, 2012).

Manuscript received November 2, 2014 accepted February 18, 2015

Manuel Delgado and Antonio SuÁrez

Dpto. de Ecuaciones Diferenciales y Análisis Numérico

Fac. de Matemáticas

Univ. de Sevilla

C. Tarfia s/n, 41012 - Sevilla, SPAIN

E-mail address: madelgado@us.es and suarez@us.es

Giovany M. Figueiredo

Universidade Federal do Pará

Faculdade de Matemática

CEP: 66075-110 Belém - Pa, BRAZIL

E-mail address: giovany@ufpa.br

Marcos T.O. Pimenta

Departamento de Matemática e Computação

Faculdade de Ciências e Tecnologia - Unesp

CEP: 19060-900, Presidente Prudente-SP, BRAZIL

E-mail address: pimenta@fct.unesp.br 